\title{
Ablación de arritmias cardiacas empleando un sistema de mapeo electroanatómico tridimensional en el Instituto Nacional Cardiovascular - INCOR
}

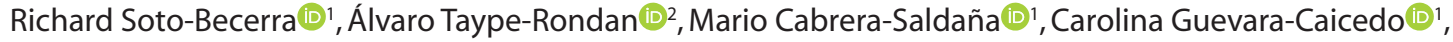

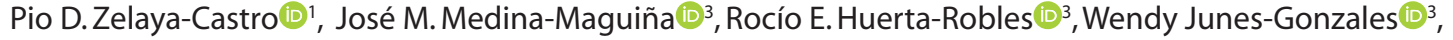
Ofelia Aráoz-Tarco (1) ${ }^{4}$, Alejandro Sangines-Montes (iD) ${ }^{5}$, Ricardo Zegarra-Carhuas (iD)
\end{abstract}

Recibido: 22 de julio 2021 Aceptado: 31 de agosto 202

\section{Filiación de los autores} Unidad de Arritmias, Instituto Nacional Cardiovascular INCOR, EsSalud, Lima, Perú

Unidad de Investigación para la Generación y Síntesis de Evidencias en Salud, Universidad San Ignacio de Loyola, Lima, Perú. Servicio de Cardiología clínica, Instituto Nacional Cardiovascula INCOR, EsSalud, Lima, Perú.

Universidad Peruana Cayetano

Heredia, Lima. Perú.

Executive Clinical Account

Specialist Biosense Webster Inc. California, USA.

\section{*Correspondencia}

Richard Soto-Becerra

Jirón Coronel Zegarra 417

Jesús María, Código postal: 15072

\section{Correo}

cardsot@gmail.com

Financiamiento

El estudio ha sido autofinanciado por los autores.

Conflictos de interés

Alejando Sanguines-Montes recibe honorarios como especialista clínico de Biosense Webster Inc. El resto de los autores declaran no tener conflictos de interés con respecto conflictos de in

Citar como:

Soto-Becerra R; Taype-Rondan A Cabrera-Saldaña M, Guevara-Caicedo C, Zelaya-Castro PD, MedinaMaguiña JM, et al. Ablación de arritmias cardiacas empleando un istema de mape electroando un sidema de mapeo electroanatón . nacional cardiovascular-INCOR. Arch Peru Cardiol Cir Cardiovasc. 2021;2(3):150-158. doi: 10.47487/ apcyccv.v2i3.147.

\section{RESUMEN}

Objetivo. Describir la experiencia inicial en ablación de arritmias cardiacas empleando mapeo 3D en el Instituto Nacional Cardiovascular INCOR (Lima-Perú). Materiales y métodos. Estudio descriptivo, retrospectivo, donde se recolectaron datos de historias clínicas de todos los pacientes en los cuales se realizó ablación empleando mapeo 3D, desde julio de 2017 a diciembre de 2019. Este procedimiento se realizó a pacientes sintomáticos y refractarios a terapia antiarrítmica. Resultados. Se recolectaron datos de 123 pacientes (mediana de edad: 46 años, 64,2\% varones), con una mediana del tiempo de enfermedad de 6 años. Entre las arritmias tratadas 19\% tuvieron fibrilación auricular; 17,5\% taquicardia auricular; $17,5 \%$ arritmias ventriculares idiopáticas; $16,6 \%$ síndrome de Wolf Parkinson White/taquicardia por reentrada auriculoventricular; $11,1 \%$ arritmias ventriculares del sistema de conducción His-Purkinje; 9,5\% taquicardia ventricular asociada con cicatriz; $6,4 \%$ flutter atrial y $2,4 \%$ taquicardia intranodal. La mediana del tiempo de fluoroscopía fue de $26 \mathrm{~min}$. La ablación tuvo éxito agudo en el 95,9\% de los casos; las complicaciones agudas se observaron en 4,8\%, y la sobrevida libre de recurrencias durante el primer año de seguimiento fue de $74 \%$. Conclusiones. Nuestra experiencia en ablación de arritmias cardiacas empleando mapeo 3D presentó una alta frecuencia de ablación exitosa aguda y baja frecuencia de complicaciones, así como una sobrevida libre de recurrencias del $74 \%$, estimada al año de seguimiento.

Palabras clave: Arritmia cardiaca; Ablación con catéter; Perú (fuente: DeCS BIREME).

\section{ABSTRACT}

\section{Ablation of cardiac arrhythmias using a three-dimensional electro- anatomical mapping system in the Instituto Nacional Cardiovascular - \\ INCOR}

Objective. To describe the initial experience in ablation of cardiac arrhythmias using 3D mapping at the Instituto Nacional Cardiovascular INCOR (Lima, Peru). Methods. A retrospective descriptive study was carried out. During February 2020, data was collected from the medical records of all patients in whom ablation was performed using 3D mapping from July 2017 to December 2019. This procedure was performed in patients with symptomatic arrhythmia refractory to antiarrhythmic therapy. Results. Data were collected from 123 patients (median age: 46 years, $64.2 \%$ male), who had a median time of illness of 6 years. Among the arrhythmias treated, 19\% had atrial fibrillation, $17.5 \%$ atrial tachycardia, $17.5 \%$ idiopathic ventricular arrhythmias, $16.6 \%$ Wolf Parkinson White syndrome / Atrioventricular reentrant tachycardia, $11.1 \%$ ventricular arrhythmias of the His-Purkinje conduction system, $9.5 \%$ scar related ventricular tachycardia associated, $6.4 \%$ atrial flutter and $2.4 \%$ intranodal tachycardia. The median fluoroscopy time was 26 minutes. Ablation was acutely successful in $95.9 \%$ of cases, acute complications were observed in $4.8 \%$, and recurrence-free survival during the first year of follow-up was $74 \%$. Conclusions. Our experience in ablation of cardiac arrhythmias using 3D mapping had a high acute success rate, low frequency of complications, and one-year recurrence-free survival of $74 \%$.

Keywords: Cardiac arrhythmia; Catheter ablation; Peru (source: MeSH NLM). 


\section{Introducción}

El tratamiento de las arritmias cardiacas ha presentado importantes avances en los últimos 40 años ${ }^{(1-4)}$. La ablación es un procedimiento invasivo percutáneo, que comprende el ingreso de catéteres al corazón. Estos procedimientos inicialmente fueron guiados de forma convencional por fluoroscopía, permitiendo mapear, registrar la actividad eléctrica endocárdica y realizar maniobras para entender el mecanismo y localizar el origen o la zona crítica de las arritmias. La ablación convencional guiada solo por fluoroscopía es usada para el tratamiento de diferentes arritmias; sin embargo, presenta limitaciones en el tratamiento de arritmias cardiacas complejas como el flutter auricular (FLA) atípico, taquicardia auricular (TA), fibrilación auricular (FA) y arritmias ventriculares ${ }^{(5)}$. En este escenario, la ablación con radiofrecuencia empleando sistema de mapeo electroanatómico tridimensional (mapeo 3D) emerge como una alternativa que puede tener ventajas frente a las estrategias farmacológicas o invasivas convencionales ${ }^{(5)}$. El sistema de mapeo 3D emite campos magnéticos de baja intensidad o campos eléctricos generadores de una gradiente de voltaje axial, esto permite detectar la posición espacial del catéter de mapeo, el cual tiene integrado un sensor en su punta que adquiere información eléctrica en cada punto de contacto entre el catéter y el tejido miocárdico, la cual es registrada en forma de electrogramas que luego son analizados para construir mapas electroanatómicos de voltaje, activación y propagación. Estos mapas, junto con las maniobras y el análisis de los electrogramas, ayudan al especialista a entender con mayor precisión el mecanismo, presencia de sustrato arrítmico y la localización de la zona critica de la arritmia cardiaca ${ }^{(6)}$.

Las evidencias sugieren que la ablación con radiofrecuencia empleando mapeo 3D puede ser una alternativa eficaz y segura en el manejo de las arritmias cardiacas ${ }^{(7)}$, debido a su adecuada reproducibilidad en estudios experimentales con animales ${ }^{(8)}$, y la menor recurrencia de arritmias auriculares en comparación con drogas antiarrítmicas en pacientes con fibrilación auricular encontrada en estudios clínicos aleatorizados ${ }^{(9)}$. Este método se ha expandido en diferentes países, de manera que el 2015 su uso se extendía al 49\% de los centros especializados en Latinoamérica ${ }^{(10)}$. En Perú, el Instituto Nacional Cardiovascular (INCOR) inició su experiencia en ablación con radiofrecuencia empleando mapeo 3D en julio de 2017, y es actualmente el centro con mayor experiencia y número de procedimientos realizados en el país.

Debido a que este método es una herramienta potencialmente útil para lograr solucionar un gran grupo de arritmias en nuestra población, y como no se han publicado reportes sobre su uso en nuestro medio, realizamos el presente estudio con el objetivo de describir las características y los resultados obtenidos en los pacientes que fueron sometidos a ablación de arritmias cardiacas empleando mapeo 3D en el Instituto Nacional Cardiovascular INCOR del Perú, en el periodo de julio 2017 a diciembre 2019.

\section{Materiales y métodos}

\section{Diseño y población}

Estudio descriptivo, retrospectivo, que incluyó a pacientes sometidos a ablación con radiofrecuencia empleando mapeo electro anatómico 3D para tratar arritmias cardiacas en el Instituto Nacional Cardiovascular INCOR (Lima, Perú) desde que comenzó a realizarse dicho procedimiento (julio del 2017) hasta diciembre del 2019. Se excluyeron aquellos pacientes con arritmias focales en quienes no se logró inducir la arritmia clínica durante el procedimiento.

\section{Recolección de variables}

Se realizó una revisión de las historias clínicas a inicios de febrero de 2020; los datos de interés se consignaron en una ficha de recolección previamente elaborada. En la ficha se registraron las características clínicas, epidemiológicas, del procedimiento realizado, el resultado final de la ablación con radiofrecuencia empleando mapeo 3D, las complicaciones y el último seguimiento clínico. Asimismo, se registró el éxito agudo de la ablación, cuya definición dependió del tipo de arritmia, como se detalla a continuación:

- Fibrilación auricular: el aislamiento eléctrico alcanzando el bloqueo de entrada y salida entre las venas pulmonares y la aurícula izquierda, y la no inducción de FA mediante sobreestimulación atrial ${ }^{(11)}$.

- Flutter auricular: el bloqueo bidireccional de la línea de ablación sobre el istmo crítico del circuito de macroreentrada y la no inducción del FLA mediante sobreestimulación atrial (12).

- Arritmias auriculares y ventriculares focales: la aplicación de radiofrecuencia (RF) en la zona de origen con la eliminación de la arritmia focal y la no inducción mediante maniobras de sobreestimulación ${ }^{(13)}$.

- Taquicardia ventricular (TV) asociado con cicatriz: la modulación del sustrato arrítmico mediante diferentes técnicas como la homogenización de la cicatriz ventricular, ablación de potenciales tardíos fragmentados o el istmo del circuito de reentrada de la TV y la no inducción de la TV monomórfica mediante maniobras de sobreestimulación ventricular ${ }^{(14)}$.

- Síndrome de Wolf- Parkinson-White o Taquicardia por reentrada atrioventricular (TRAV): la eliminación de la vía accesoria durante la aplicación de RF, documentada a través de la perdida de preexcitación ventricular y la disociación ventrículo-auricular ${ }^{(15)}$. 
- Taquicardia por reentrada intranodal (TRIN): la aplicación de RF sobre la vía lenta del nodo aurículo-ventricular con presencia de latidos nodales durante la aplicación de RF, no evidenciando dos o más ecos nodales ni inducción de la taquiarritmia durante la sobreestimulación posablación ${ }^{(16)}$.

Las complicaciones agudas fueron definidas como aquellas ocurridas en el periprocedimiento o dentro de las $24 \mathrm{~h}$ posprocedimiento ${ }^{(17)}$. Las complicaciones mayores fueron definidas como aquellos eventos adversos que, según la opinión del especialista tratante, estuvieron relacionados al procedimiento de ablación y que necesitaron de una intervención para su tratamiento, causando mayor tiempo de recuperación y mayor tiempo de hospitalización ${ }^{(18)}$.

La recurrencia fue definida como la reaparición de la arritmia clínica tratada después de la ablación. En los pacientes con posablación de FA/FLA, definimos recurrencia a la reaparición de la arritmia clínica pasado los 3 meses posablación, estas tenían que estar reportadas en la historia clínica mediante el registro electrocardiográfico o estudio Holter.

El presente estudio fue aprobado por el Comité de Ética del Instituto Nacional Cardiovascular INCOR, antes de su ejecución. No se recolectaron variables que permitan identificar a los pacientes.

\section{Descripción del procedimiento de ablación}

En los pacientes con arritmias complejas, sintomáticos y refractarios a terapia antiarrítmica, se realizaron las ablaciones con radiofrecuencia empleando mapeo 3D con el sistema CARTO 3 (Biosense Webster, Johnson \& Johnson) y catéteres de ablación irrigados de 3,5 mm (Navistar Thermocool, Biosense Webster, Johnson \& Johnson), con una distancia inter-electrodo punta anillo de $1 \mathrm{~mm}$. Los electrogramas bipolares fueron registrados entre los pares de electrodos distales y proximales, filtrados entre 16 a $500 \mathrm{~Hz}$. Los electrogramas unipolares fueron registrados entre cada electrodo del catéter de ablación y la central terminal de Wilson, filtrados entre 2 a $240 \mathrm{~Hz}$.

Los procedimientos fueron realizados en una sala de intervencionismo cardiovascular, bajo sedación o anestesia general. Los pacientes bajo anestesia general fueron aquellos cuyo procedimiento necesitó de un abordaje transeptal guiado por ecocardiografía transesofágica o un abordaje epicárdico siguiendo la técnica descrita por Sosa et al. ${ }^{(19)}$.

Según el tipo de arritmia se procedía a ingresar diferentes catéteres diagnósticos y protocolos de estimulación para la inducción y entendimiento del mecanismo de la taquiarritmia. Posteriormente, se ingresaba el catéter de ablación para la reconstrucción anatómica 3D, mapa de activación, propagación, mapa de voltaje unipolar y bipolar, según el tipo de arritmia. La aplicación de radiofrecuencia en la zona crítica fue titulada con diferentes valores de poder, de acuerdo con el criterio del operador. Una vez alcanzado el resultado deseado para cada tipo de arritmia, se consideró éxito agudo a la ausencia de recurrencia de la arritmia clínica dentro de los 30 min de observación (20).

El seguimiento clínico se realizó dentro de la primera semana posprocedimiento, luego al primer mes, al tercer mes, al sexto mes y cada año, e incluyó consulta presencial o telefónica, toma de electrocardiograma y estudio Holter de $24 \mathrm{~h}$.

\section{Análisis estadístico}

El análisis estadístico se realizó empleando el paquete Stata v15. Se realizó un análisis univariado utilizando medidas de tendencia central, medidas de dispersión, frecuencias absolutas y frecuencias relativas. Se analizó la sobrevida libre de recurrencias general empleando la curva de Kaplan Meier.

\section{Resultados}

Entre julio del 2017 y diciembre del 2019, en el INCOR se sometió a 133 pacientes a ablación con radiofrecuencia empleando mapeo 3D, para tratar arritmias cardiacas. Se excluyeron diez pacientes con arritmias focales en quienes no se logró inducir la arritmia clínica durante el procedimiento; finalmente, se analizaron los datos de 123 pacientes.

La mediana de edad de los pacientes fue de 46 años; $64,2 \%$ fueron varones, y la mediana del tiempo de enfermedad fue de 6 años. Todos los pacientes fueron sintomáticos, siendo las palpitaciones la forma más frecuente de presentación (93,5\%). Todos fueron refractarios a tratamiento farmacológico, y el 86,9\% registró ausencia de cardiopatía estructural. El total de arritmias tratadas fueron 126, la frecuencia y el tipo de arritmia son presentados en la (Figura 1). Las características de la población, de la intervención, abordaje, tipo de ablación y desenlaces se describen en la (Tabla 1 y 2).

De 24 pacientes con FA, 20 fueron paroxísticas y 4 persistentes; el end-point fue aislar eléctricamente las cuatro venas pulmonares de la aurícula izquierda mediante un aislamiento antral circunferencial punto a punto, consiguiéndolo en el 95.8\%. En 4 pacientes fue necesario realizar líneas de ablación en la pared posterior de la aurícula izquierda, vena cava superior o istmo cavo tricuspideo, la recurrencia fue del 33,3\% y las complicaciones alcanzaron el 8,3\%.

De los 22 pacientes con arritmias ventriculares idiopáticas, 11 estuvieron localizadas en el tracto de salida del ventrículo derecho, y 4 en la región infundibular. La mediana de la carga 


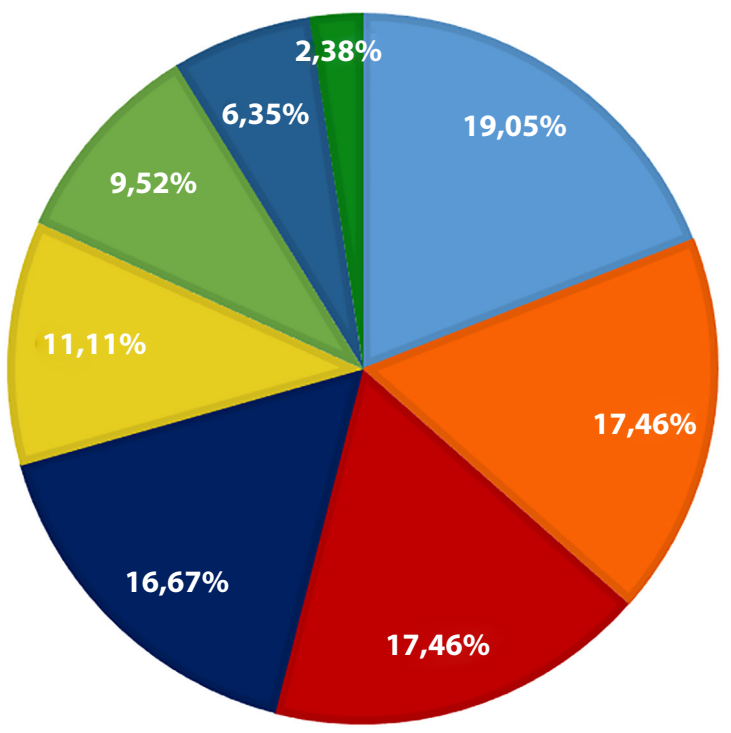

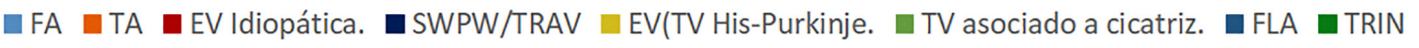

\begin{tabular}{|c|c|c|c|c|c|c|c|}
\hline FA $^{1}$ & TA $^{2}$ & EV $^{3}$ Idiopática. & SWPW/TRAV & EV(TV His-Purkinje. & TV $^{4}$ asociado a cicatriz. & FLA $^{5}$ & TRIN $^{6}$ \\
\hline 24 & 22 & 22 & 21 & 14 & 12 & 8 & 3 \\
\hline
\end{tabular}

Figura 1. Arritmias tratadas con sistema de mapeo electroanatómico 3D en INCOR ( $N=126)$

Frecuencia y porcentaje de arritmias clínicas tratadas mediante ablación con sistema electroanatómico 3D, FA: fibrilación auricular. TA: taquicardia auricular EV: extrasístole ventricular. SWPW/TRAV: síndrome de Wolf- Parkinson-White/Taquicardia por reentrada atrioventricular. TV: taquicardia ventricular. FLA: flutter auricular. TRIN: taquicardia por reentrada intranodal..

arrítmica fue del $32 \%$ y se registró éxito agudo en la ablación en el $95.5 \%$ de casos con una recurrencia del $23.8 \%$.

Catorce pacientes tuvieron arritmias ventriculares asociadas al sistema de conducción His-purkinje, 13 fueron TV fascicular posterior izquierda y uno tuvo TV hisiana. Todos presentaron éxito agudo en la ablación, documentando recurrencia posablación del 7,1\%. Los 12 pacientes con ablación de taquicardia ventricular asociado a cicatriz presentaban una cardiopatía estructural de base, siendo la cardiopatía isquémica la más frecuente $(50 \%)$, seguida de la cardiomiopatía dilatada idiopática (33.3\%) y la cardiomiopatía arritmogénica del ventrículo derecho (16.7\%), en estos casos la ablación fue exitosa en $91.6 \%$ con una recurrencia en el $25 \%$. Realizamos abordaje epicárdico en cuatro casos, debido a recurrencia posablación y falla de la ablación endocárdica. El abordaje endoepicárdico fue realizado solo en pacientes con cardiomiopatía no isquémica (3 dilatada idiopática y 1 con cardiomiopatía arritmogénica del ventrículo derecho).

En general el $95,9 \%$ de los pacientes sometidos a ablación con radiofrecuencia empleando mapeo 3D (Figura 2) presentaron éxito agudo y el 4,8\% presentaron alguna complicación. La sobrevida libre de recurrencia arrítmica estimada al año fue de 74\% (Figura 3).

\section{Discusión}

Nuestra experiencia inicial en la ablación de arritmias cardiacas empleando mapeo electro anatómico 3D, encontró una alta tasa de ablación exitosa aguda y baja frecuencia de complicaciones. La ablación de arritmias cardiacas con radiofrecuencia empleando sistema de mapeo 3D se ha convertido en una herramienta terapéutica común para el manejo de arritmias cardiacas complejas (Figura 4). Esta técnica permite definir con mayor precisión la anatomía y el mecanismo de la arritmia y presenta ventajas frente a otros métodos, por lo que se ha convertido en una estrategia invasiva extensamente usada para diferentes tipos de arritmias cardiacas ${ }^{(7,9)}$.

El tiempo de enfermedad prolongado hasta llegar al procedimiento que encontramos en el estudio, se debe principalmente a la reciente incorporación de esta tecnología en nuestro país. La indicación de este procedimiento se basó en guías internacionales y de nuestra institución (21), priorizando la presencia de síntomas y la refractariedad a terapia antiarrítmica. El éxito agudo encontrado fue similar al descrito en diferentes registros internacionales, que reportan frecuencias de éxito agudo entre el 91 a $95,4 \%{ }^{(10,22,23)}$. 
Tabla 1. Características clínicas de los pacientes que fueron a ablación con un sistema de mapeo 3D en INCOR $(\mathrm{N}=123)$

\begin{tabular}{lc}
\hline Características clínicas & n (\%) \\
\hline Edad en años - mediana (RIC) & $46(24-62)$ \\
Sexo masculino & $79(64,2)$ \\
Tiempo de enfermedad en años - mediana (RIC) & $6(2-10)$ \\
Síntomas cardiovasculares & \\
$\quad$ Palpitaciones & $115(93,5)$ \\
$\quad$ Disnea & $22(17,8)$ \\
\multicolumn{1}{l}{ Síncope } & $6(4,8)$ \\
\hline \multicolumn{1}{c}{ Dolor torácico } & $19(15,4)$
\end{tabular}

\section{Terapia farmacológica}

$\begin{array}{lc}\text { Betabloqueadores } & 61(49,5) \\ \text { Bloqueadores canales de } \mathrm{Ca}++ & 19(15,4) \\ \text { Digoxina } & 1(0,81) \\ \text { Propafenona } & 45(36,5) \\ \text { Amiodarona } & 45(36,5)\end{array}$

\section{Comorbilidades asociadas}

Hipertensión arterial $\quad 16(13,0)$

Diabetes mellitus

Cardiopatía isquémica

$7(5,6)$

Evento cerebrovascular

\section{Cardiopatía estructura}

Ninguna

$107(86,9)$

CMPD isquémica

CMPD idiopática

CAVD

Anomalía de Ebstein

Fracción de eyección en \% - mediana (RIC)

Ablaciones previas

RIC: rango intercuartil. CMPD: cardiomiopatía dilatada. CAVD: cardiomiopatía arritmogénica del ventrículo derecho.

Nuestra experiencia muestra que la ablación de FA registra una recurrencia de $33 \%$, los estudios que evalúan la eficacia y seguridad de la ablación de la FA, usando tecnología comparable a nuestro centro, reportaron una sobrevida libre de recurrencia entre el 63 al 86\% a los 9- 12 meses de seguimiento en FA paroxística ${ }^{(24,25)}$, de 60 a $74 \%$ en FA persistente ${ }^{(26,27)}$ con una tasa de complicaciones mayores del $4,5 \%$ y mortalidad de $0,15 \%{ }^{(28)}$.

En los pacientes con arritmias ventriculares idiopáticas registramos una tasa de éxito similar a otros registros que muestran una tasa de éxito agudo del 85 al 90\%, con una sobrevida libre de recurrencia a largo plazo del $75,80 \%$ y una tasa de complicaciones del $2 \%{ }^{(29,30)}$. La incorporación de nuevas tecnologías permitirá mejorar nuestra eficacia y seguridad y a su vez abordar con mejores resultados a las arritmias de
Tabla 2. Características del procedimiento

\begin{tabular}{|c|c|}
\hline Características de la intervención & Mediana (RIC) \\
\hline $\begin{array}{l}\text { Tiempo de procedimiento en } \\
\text { minutos. }\end{array}$ & $225(180-300)$ \\
\hline Tiempo de fluoroscopía en minutos. & $26(14-37)$ \\
\hline Dosis de Radiación en mGy. & $470(165-1092)$ \\
\hline $\begin{array}{l}\text { Tiempo de radiofrecuencia en } \\
\text { segundos. }\end{array}$ & $684(317-1351)$ \\
\hline Puntos de radiofrecuencia. & $29(12-97)$ \\
\hline Tipo de abordaje realizado & $\mathrm{n}(\%)$ \\
\hline Derecho & $52(42,0)$ \\
\hline Transeptal & $38(30,8)$ \\
\hline Retroaórtico & $29(23,5)$ \\
\hline Endoepicárdico & $4(3,2)$ \\
\hline Tipo de ablación realizada & $\mathrm{n}(\%)$ \\
\hline Focal & $66(53,6)$ \\
\hline Aislamiento & $28(22,7)$ \\
\hline Modulación de sustrato & $9(7,3)$ \\
\hline Lineal & $20(16,2)$ \\
\hline Desenlaces & $\mathrm{n}(\%)$ \\
\hline Ablación con éxito agudo & $118(95,9)$ \\
\hline Complicaciones agudas & $6(4,8)$ \\
\hline Complicaciones vasculares & $3(2,4)$ \\
\hline Derrame pericárdico & $2(1,6)$ \\
\hline Mortalidad & 0 \\
\hline
\end{tabular}

RIC: rango intercuartil.

localización más complejas entre ellas LV Summit, musculo papilar o banda moderadora.

Las arritmias ventriculares del sistema de conducción His Purkinje reportada en nuestro estudio estuvo compuesto principalmente por taquicardia ventricular fascicular posterior izquierda. Registramos una tasa de éxito similar a otros reportes de ablación de TV fascicular (éxito del 100\%) que reportan sobrevida libre de recurrencia del $85 \%$ a los 4,5 años de seguimiento ${ }^{(31)}$.

Los estudios clínicos sobre ablación de TV recurrente en pacientes posinfarto muestran una sobrevida libre de recurrencias a los 6 meses del 54\% y una tasa de mortalidad del 18\% al año de seguimiento ${ }^{(32)}$. Los estudios de ablación de TV en pacientes con cardiopatía no isquemia presentan grupos muy heterogéneos, suelen ser observacionales y de un solo centro, presentan tasas de éxito agudo entre el 38 al 74\% y tasas de recurrencia entre el 29 al $58 \%$ con una media de seguimiento de 9 a 22 meses ${ }^{(33-35)}$.

No registramos mortalidad asociada con el procedimiento. El 4,8\% presentaron complicaciones agudas que en su mayoría 


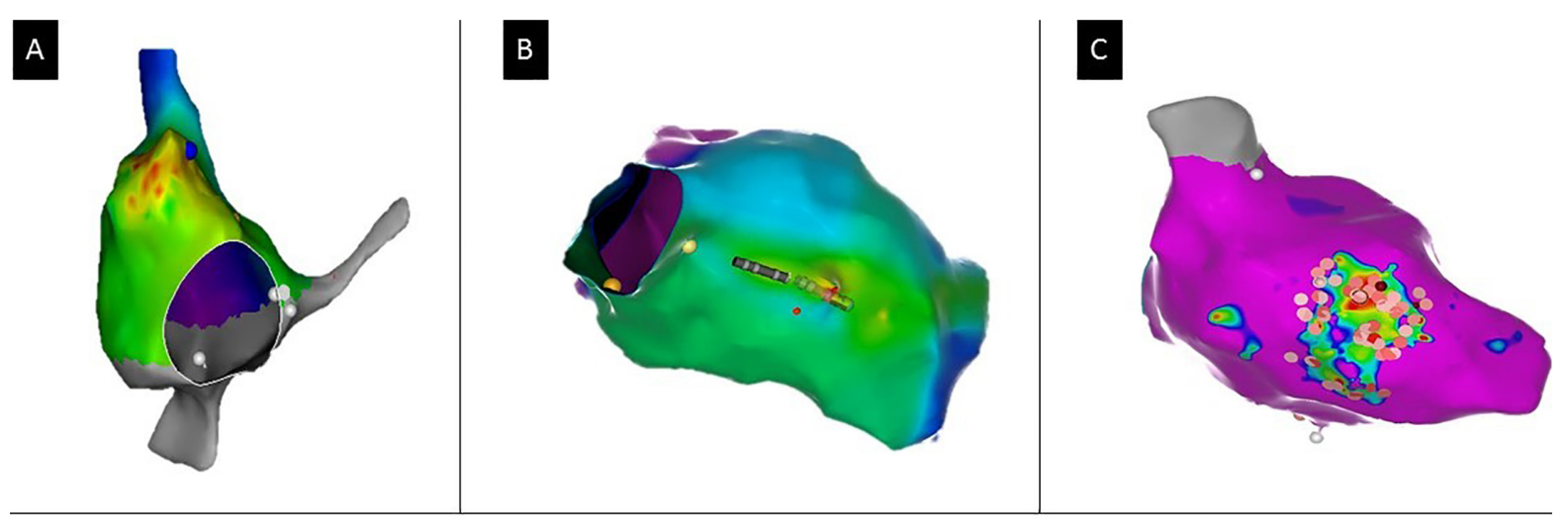

D
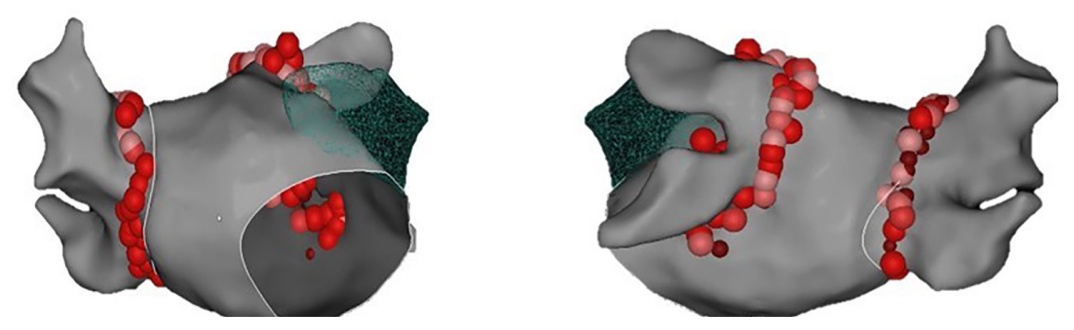

Figura 2. Mapa electroanatómico 3D para ablación de arritmias complejas en el INCOR

A. Mapa de activación en taquicardia atrial focal de orejuela derecha. B. Mapa de activación en extrasístole ventricular focal del musculo papilar posterior del ventrículo izquierdo. C. Modulación de sustrato arrítmico para taquicardia ventricular asociado a cicatriz definido mediante mapa de voltaje bipolar con áreas de bajo voltaje en región medio septal $\mathrm{D}$. aislamiento eléctrico antral circunferencial de venas pulmonares.

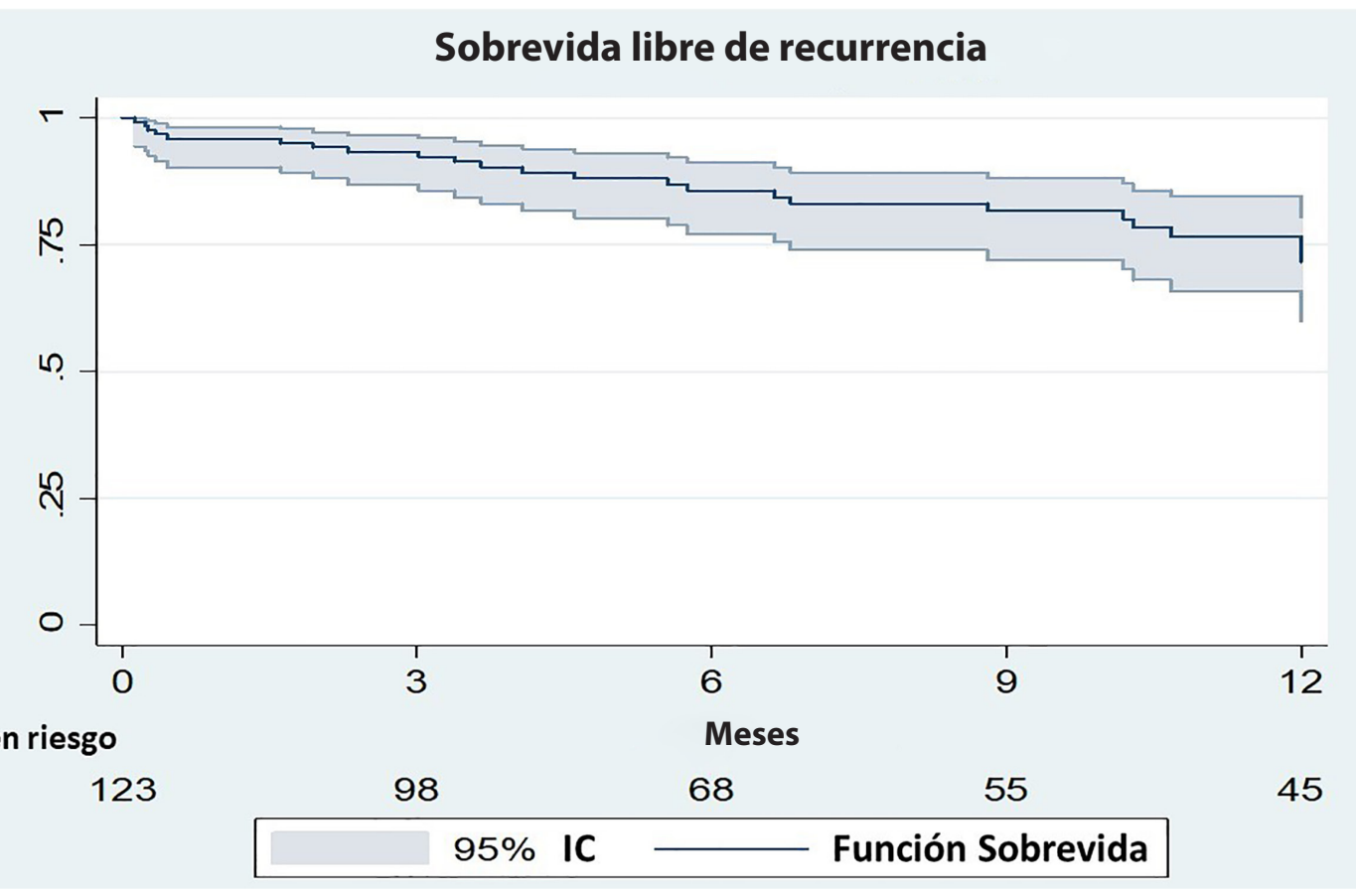

Figura 3. Sobrevida libre de recurrencias de los pacientes que fueron a ablación con sistema de mapeo electroanatómico $3 \mathrm{D}$ en INCOR $(\mathrm{N}=123)$

Sobrevida estimada libre de recurrencias empleando Kaplan Meier en el seguimiento clínico de los pacientes con éxito agudo posprocedimiento, al año de seguimiento se estima que el $74 \%$ está libre de recurrencias. 
Figura 4. Imagen central: Ablación de arritmias cardiacas empleando un sistema de mapeo electroanatómico tridimensional en el Instituto Nacional Cardiovascular - INCOR

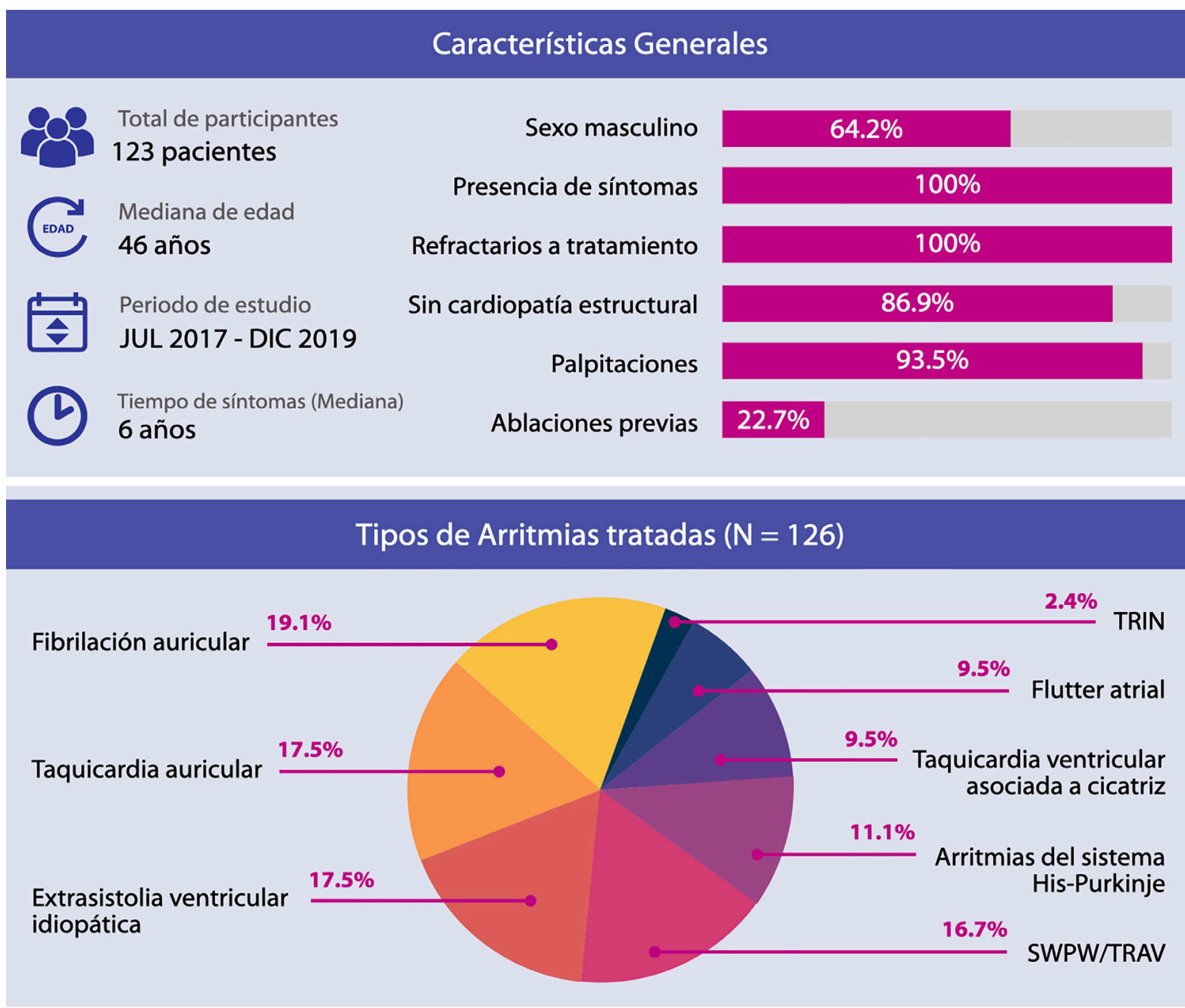

\section{Resultados}

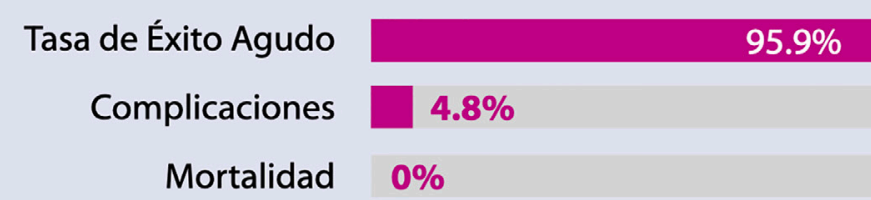

Sobrevida sin recurrencias al año

$74 \%$

\section{Soto-Becerra R, et al. Arch Peru Cardiol Cir Cardiovasc. 2021;2(3):150-158}

TRIN: taquicardia por re-entrada intranodal; SWPW/: síndrome de Wolf- Parkinson- White; TRAV: taquicardia por re entrada auriculo ventricular

fueron vasculares $(2,4 \%)$ seguido de derrame pericárdico con necesidad de drenaje $(1,6 \%)$, estos hallazgos son similares a otros registros que han encontrado entre 2 y $4,5 \%$, siendo las complicaciones vasculares las más frecuentes ${ }^{(10,22,23,28)}$.

Consideramos que es importante seguir recolectando datos sobre las ablaciones con radiofrecuencia empleando mapeo electro anatómico 3D en nuestro centro, dado que nos permitirá identificar variables sobre las cuales intervenir para poder mejorar nuestros resultados. La incorporación de nuevas estrategias, abordajes y nueva tecnología como los catéteres con sensores de fuerza de contacto, catéteres multielectrodos para mapeo de alta densidad, ecocardiografía intracardiaca y nuevos softwares dentro del sistema de mapeo 3D ayudará a incrementar 
la frecuencia de éxito agudo y reducir las complicaciones y recurrencias.

\section{Limitaciones y fortalezas}

Es necesario tener en cuenta algunas consideraciones del presente estudio a la hora de interpretar sus resultados: 1) Es un estudio observacional realizado mediante la revisión de historias clínicas, por lo cual se tuvo que confiar en lo registrado en dichos documentos. 2) Al momento de la recolección de datos, un grupo importante de pacientes tenían menos de 12 meses desde que fueron sometidos al procedimiento; por lo cual a los 12 meses después del procedimiento solo se tenía información de 21 pacientes con recurrencia y 45 sin recurrencia (es decir, 66 pacientes [53,7\%] de 123); lo cual puede sesgar el resultado de recurrencia, por ello se requieren futuros estudios con menor frecuencia de pérdidas. 3) Estos resultados fueron obtenidos en un centro especializado de referencia nacional, por lo que podrían no extrapolarse si es que son realizados en otros centros de menor resolución. Sin embargo, este es el primer reporte de ablación con mapeo 3D en Perú, en el que se presenta en detalle el procedimiento que se viene realizando en el INCOR. De esta manera brindamos una línea de base sobre el uso este procedimiento en nuestro medio.

\section{Conclusiones}

Nuestra experiencia inicial en pacientes con arritmia cardiaca sintomática, refractarios a terapia antiarrítmicay sometidos a ablación con radiofrecuencia empleando mapeo electro anatómico 3D en nuestra institución encontró una alta frecuencia de ablación exitosa aguda y baja frecuencia de complicaciones, así como una sobrevida libre de recurrencias del $74 \%$ estimada al año de seguimiento.

\section{Agradecimientos}

Agradecemos a la Dra. Carina Hardy y al Dr. Luis Carlos Sáenz, por su asesoría en la etapa inicial para realizar los primeros procedimientos de mapeo electroanatómico 3D en el INCOR. Asimismo, agradecemos a todo el personal de salud del Instituto Nacional Cardiovascular que haya participado en algún procedimiento de mapeo electro anatómico 3D, por su valioso aporte en la ejecución de los procedimientos.

\section{Contribuciones de los autores}

RSB, MCS, CGC, PZC y RZC plantearon la idea de investigación. RSB, JMM, RHR y WJG recolectaron los datos del estudio. RSB, OAT y ATR analizaron los datos. RSB y RZC redactaron la primera versión del manuscrito. Todos los autores participaron en la redacción y aceptaron la versión final del estudio.

\section{Referencias bibliográficas}

1. Scheinman MM, Morady F, Hess DS, Gonzales R. Catheter-induced ablation of the atrioventricular junction to control refractory supraventricular arrhythmias. JAMA. 1982;248(7):851-5.

2. Gallagher JJ, Svenson RH, Kasell JH, German LD, Bardy GH, Broughton $A$, et al. Catheter technique for closed-chest ablation of the atrioventricular conduction system. N Engl J Med. 1982;306(4):194200. doi: 10.1056/NEJM198201283060402.

3. Huang SK, Bharati S, Graham AR, Lev M, Marcus Fl, Odell RC. Closed chest catheter desiccation of the atrioventricular junction using radiofrequency energy--a new method of catheter ablation. J Am Coll Cardiol. $1987 ; 9(2): 349-58$.

4. Huang SK. Radio-frequency catheter ablation of cardiac arrhythmias: appraisal of an evolving therapeutic modality. Am Heart J. 1989 Dec;118(6):1317-23.

5. Friedman PA. Novel mapping techniques for cardiacelectrophysiology. Heart. 2002 Jun;87(6):575-82. doi: 10.1136/heart.87.6.575.

6. Duru F.CARTO three-dimensional non-fluoroscopic electroanatomic mapping for catheter ablation of arrhythmias: a useful tool or an expensive toy for the electrophysiologist? Anadolu Kardiyol Derg. 2002;2(4):330-7.

7. Kim YH, Chen SA, Ernst S, Guzman CE, Han S, Kalarus Z, et al. 2019 APHRS expert consensus statement on three-dimensional mapping systems for tachycardia developed in collaboration with HRS, EHRA, and LAHRS. J Arrhythm. 2020;36(2):215-70. doi: 10.1002/joa3.12308.
8. Gepstein L, Hayam G, Ben-Haim SA. A novel method for nonfluoroscopic catheter-based electroanatomical mapping of the heart. In vitro and in vivo accuracy results. Circulation. 1997;95(6):1611-22. doi: 10.1161/01.cir.95.6.1611.

9. Asad ZUA, Yousif A, Khan MS, Al-Khatib SM, Stavrakis S. Catheter Ablation Versus Medical Therapy for Atrial Fibrillation: A Systematic Review and Meta-Analysis of Randomized Controlled Trials. Circ Arrhythm Electrophysiol. 2019;12(9):e007414. doi: 10.1161/ CIRCEP.119.007414.

10. Keegan R, Aguinaga L, Fenelon G, Uribe W, Rodriguez G, Scanavacca $\mathrm{M}$, et al. The first Latin American Catheter Ablation Registry. Europace. 2015;17(5):794-800. doi: 10.1093/europace/euu322.

11. Pappone C, Rosanio S, Oreto G, Tocchi M, Gugliota F, Vicedomini G, et al. Circumferential radiofrequency ablation of pulmonary vein ostia: A new anatomic approach for curing atrial fibrillation. Circulation. 2000;102(21):2619-28. doi: 10.1161/01.cir.102.21.2619.

12. Peyrol M, Sbragia P. Typical Atrial Flutter - When Do You Say You Have Got It. J Atr Fibrillation. 2012 Oct 6;5(3):712. doi: 10.1371/jafib.712.

13. Zhong L, Lee $\mathrm{YH}$, Huang $\mathrm{XM}$, Asirvatham $\mathrm{S}$, Shen $\mathrm{W}$, Friedman $\mathrm{P}$, et al. Relative efficacy of catheter ablation vs antiarrhythmic drugs in treating premature ventricular contractions: a single-center retrospective study. Heart Rhythm. 2014 Feb;11(2):187-93. doi: 10.1016/j.hrthm.2013.10.033.

14. Di Biase L, Santangeli P, Burkhardt DJ, Bai R, Mohanty P, Carbucicchio $C$, et al. Endo-epicardial homogenization of the scar versus 
limited substrate ablation for the treatment of electrical storms in patients with ischemic cardiomyopathy. J Am Coll Cardiol. 2012 Jul 10;60(2):132-41. doi: 10.1016/j.jacc.2012.03.044.

15. Gulletta S, Tsiachris D, Della Bella P. Catheter ablation of an anteroseptal accessory pathway guided by contact force monitoring technology and precise electroanatomical mapping. Europace. 2014 Jun;16(6):825. doi: 10.1093/europace/eut254.

16. Katritsis DG, Josephson ME. Classification, Electrophysiological Features and Therapy of Atrioventricular Nodal Reentrant Tachycardia. Arrhythm Electrophysiol Rev. 2016;5(2):130-5. doi:10.15420/AER.2016.18.2.

17. Aksu T, Yalin K, Guler TE, Bozyel S, Heeger C. Tilz R. Acute Procedural Complications of Cryoballoon Ablation: A Comprehensive Review.J Atr Fibrillation. 2019;12(3):2208. Published 2019 Oct 31. doi:10.4022/ jafib.2208.

18. Bohnen M, Stevenson WG, Tedrow UB, Michaud G, John R, Epstein $L$, et al. Incidence and predictors of major complications from contemporary catheter ablation to treat cardiac arrhythmias. Heart Rhythm. 2011 Nov;8(11):1661-6. doi: 10.1016/j.hrthm.2011.05.017.

19. Sosa E, Scanavacca M, D'Avila A, Piccioni J, Sanchez O, Velarde JL, et al. Endocardial and epicardial ablation guided by nonsurgical transthoracic epicardial mapping to treat recurrent ventricular tachycardia. J Cardiovasc Electrophysiol.1998;9:229-39. doi: 10.1111/j.1540-8167.1998.tb00907.x.

20. Gurevitz OT, Glikson M, Asirvatham S, Kester T, Grice S, Munger T, et al. Use of advanced mapping systems to guide ablation in complex cases: experience with noncontact mapping and electroanatomic mapping systems. Pacing Clin Electrophysiol. 2005; 28: 316- 23. doi:10.1111/j.1540-8159.2005.09477.x.

21. Soto-Becerra R, Zafra-Tanaka JH, Goicochea-Lugo S, Alarcon-Ruiz C, Pacheco-Barrios K, Taype-Rondan A, et al. Guía de práctica clínica para el manejo de pacientes con fibrilación auricular en el Seguro Social del Perú (EsSalud). An Fac Med.2019;80 (2):250-63.

22. Holmqvist $F$, Kesek $M$, Englund A, Blomstrom-Lundqvist $C$, Karlsson $L$, Kenneback $G$, et al. A decade of catheter ablation of cardiac arrhythmias in Sweden: ablation practices and outcomes. Eur Heart J. 2019 Mar 7;40(10):820-30. doi: 10.1093/eurheartj/ehy709.

23. Ibáñez Criado JL, Quesada A, Cózar R. Spanish Catheter Ablation Registry. 18th Official Report of the Spanish Society of Cardiology Working Group on Electrophysiology and Arrhythmias. Rev Esp Cardiol (Engl Ed). 2019 Dec;72(12):1031-42. English, Spanish. doi: 10.1016/j.rec.2019.08.005.

24. Wilber DJ, Pappone C, Neuzil P, De Paola A, Marchlinski F, Natale A, et al. Comparison of antiarrhythmic drug therapy and radiofrequency catheter ablation in patients with paroxysmal atrial fibrillation: a randomized controlled trial. JAMA. 2010 Jan 27;303(4):333-40. doi: 10.1001/jama.2009.2029.
25. Pappone C, Augello G, Sala S, Gugliotta F, Vicedomini G, Gulleta S, et al. A randomized trial of circumferential pulmonary vein ablation versus antiarrhythmic drug therapy in paroxysmal atrial fibrillation: the APAF Study. J Am Coll Cardiol. 2006 Dec 5;48(11):2340-7. doi: 10.1016/j. jacc.2006.08.037.

26. Oral H, Pappone C, Chugh A, Good E, Bogun F, Pelosi Jr F, et al. Circumferential pulmonary-vein ablation for chronic atrial fibrillation. NEngl J Med. 2006 Mar 2;354(9):934-41. doi: 10.1056/NEJMoa050955.

27. MontL, Bisbal F, Hernández-Madrid A, Pérez-CastellanoN, Viñolas X, Arenal $A$, et al. Catheter ablation vs. antiarrhythmic drug treatment of persistent atrial fibrillation: a multicentre, randomized, controlled trial (SARA study). Eur Heart J. 2014 Feb;35(8):501-7. doi: 10.1093/eurheartj/eht457.

28. Cappato R, Calkins H, Chen SA, Davies W, lesaka Y, Kalman J, et al. Updated worldwide survey on the methods, efficacy, and safety of catheter ablation for human atrial fibrillation. Circ Arrhythm Electrophysiol. 2010 Feb;3(1):32-8. doi: 10.1161/CIRCEP.109.859116.

29. Tondo C, Carbucicchio C, Dello Russo A, majocchi B, Zucchetti M, Pizzamiglio F, et al. Idiopathic Ventricular Tachycardia:Transcatheter Ablation or Antiarrhythmic Drugs? J Atr Fibrillation. 2015;7(5):1164. doi:10.4022/jafib.1164.

30. Callans DJ. Catheter ablation of idiopathic ventricular tachycardia arising from the aortic root. J Cardiovasc Electrophysiol. 2009 Aug;20(8):969-72. doi: 10.1111/j.1540-8167.2009.01461.x.

31. Liu Y, Fang Z, Yang B, Kojodjojo P, Chen H, Ju W, et al. Catheter Ablation of Fascicular Ventricular Tachycardia: Long-Term Clinical Outcomes and Mechanisms of Recurrence. Circ Arrhythm Electrophysiol. 2015;8(6):1443-51. doi:10.1161/CIRCEP.115.003080.

32. Stevenson WG, Wilber DJ, Natale A, Jackman W, Marchlinski F, Talbert $\mathrm{T}$, et al. Irrigated radiofrequency catheter ablation guided by electroanatomic mapping for recurrent ventricular tachycardia after myocardial infarction: the multicenter thermocool ventricular tachycardia ablation trial. Circulation. 2008 Dec 16;118(25):2773-82. doi: 10.1161/CIRCULATIONAHA.108.788604.

33. Katja Zeppenfeld. Ventricular Tachycardia Ablation in Nonischemic Cardiomyopathy. JACC: Clinical Electrophysiology 2018; 4(9). doi: 10.1016/j.jacep.2018.06.014.

34. Piers S.R., Leong D.P., Van Huls van Taxis C.F, Tayyebi M, Trines S, Pijnappels D, et al. Outcome of ventricular tachycardia ablation in patients with nonischemic cardiomyopathy: the impact of noninducibility. Circ Arrhythm Electrophysiol 2013; 6:513-21. DOI: 10.1161/CIRCEP.113.000089.

35. Muser D, Santangeli P, Castro SA, Pathak R, Liang J, Hayashi T, et al. Long-Term Outcome After Catheter Ablation of Ventricular Tachycardia in Patients With Nonischemic Dilated Cardiomyopathy. Circ Arrhythm Electrophysiol. 2016 Oct;9(10):e004328. doi: 10.1161/ CIRCEP.116.004328. 